\title{
HIV Risk Perception, Sexual Behavior, and HIV Prevalence among Men-Who-Have-Sex-with-Men at a Community-Based Voluntary Counseling and Testing Center in Kuala Lumpur, Malaysia
}

\author{
Kwee Choy Koh ${ }^{1}$ and Lit Sin Yong ${ }^{2}$ \\ ${ }^{1}$ Department of Medicine, Clinical School, International Medical University, Jalan Rasah, 70300 Seremban, Negeri Sembilan, Malaysia \\ ${ }^{2}$ Department of Medicine, Hospital Tuanku Ja'afar Seremban, Jalan Rasah, 70300 Seremban, Negeri Sembilan, Malaysia
}

Correspondence should be addressed to Kwee Choy Koh; kweechoy_koh@imu.edu.my

Received 24 October 2013; Revised 10 June 2014; Accepted 12 June 2014; Published 25 June 2014

Academic Editor: Sandro Cinti

Copyright (C) 2014 K. C. Koh and L. S. Yong. This is an open access article distributed under the Creative Commons Attribution License, which permits unrestricted use, distribution, and reproduction in any medium, provided the original work is properly cited.

\begin{abstract}
We describe the HIV risk perception, sexual behavior, and HIV prevalence among 423 men-who-have-sex-with-men (MSM) clients who received voluntary counseling and testing (VCT) services at a community-based center in Kuala Lumpur, Malaysia. The mean age was 29 years old. One hundred one (23.9\%) clients rated themselves as low risk, $118(27.9 \%)$ as medium risk, 36 (8.5\%) as high risk, and 168 (39.7\%) were unsure of their risk. Twenty-four (9.4\%) clients tested HIV positive (4 (4\%) low risk, 9 (7.6\%) medium risk, 11 (30.6\%) high risk, and $13(7.7 \%)$ unsure risk). We found a positive correlation between risk perception and HIV infection in this study. Clients with high HIV risk perception have $17 x$ the odds of testing HIV positive compared to low risk clients. High HIV risk perception was significantly associated with multiple sex partners, multiple types of sex partners, alcohol use before intercourse, unprotected sex beyond 6 months, and inconsistent condom use during anal sex compared to low risk clients. There were no statistically significant differences between medium risk and unsure risk clients compared to low risk clients. Strategies should be targeted towards change in sexual practices among those who are perceived to be at high risk.
\end{abstract}

\section{Introduction}

HIV risk perception studies among men-who-have-sex-withmen (MSM) have often reported similar findings, namely: inaccurate or misperception of HIV risk being common among MSM $[1,2]$, perception of low risk being associated with no previous HIV testing and considerable high risk behavior [3-5], and moderate/high risk being associated with unrecognized HIV infection [4].

Compared to the general adult population, MSM in Asia have 18.7 times the odds of being HIV infected [6]. Across Asia, HIV prevention expenditures targeting MSM have been reported to range between $0 \%$ in China to only $4 \%$ in Thailand [7]. The lack of funding for these programs is lamentable as prevention and harm reduction strategies targeting MSM are successful in reducing high-risk behaviors $[8,9]$. In Malaysia, homosexuality is illegal and stigmatization is a problem. MSM in Malaysia are part of the marginalized most-at-risk populations (MARP) that include commercial sex workers, migrant workers, and transgender people. HIV prevention programs including HIV testing among the MARP in Malaysia are mainly spearheaded by several nongovernmental organizations (NGO) with very limited resources. We present the findings of a study where we explored the relationship between HIV risk perception in a cohort of MSM who sought VCT services at a NGO-run center in Kuala Lumpur, Malaysia, and their sexual behavior and risk factors. Our study shows that there exists a significant association between high HIV risk perception with high risk sexual behavior and HIV positivity. Targeting MSM with high 
risk perception may indeed be the strategy to consider in a resource-limited setting in order to gain maximum benefits among the MSM in Malaysia.

\section{Materials and Methods}

Data were collated from the pre-HIV testing questionnaires filled by clients seeking VCT services at a communitybased VCT centre from January 2008 to December 2008. Completion of the questionnaire was voluntary and no data identifiable to the client were required. A total of 740 clients sought VCT services at the centre between January 1, 2008, and December 31, 2008. Of these, 433 clients disclosed their sexuality as either homosexual or bisexual and were collectively categorized as MSM in this study. The profiles, sexual practices, and HIV prevalence of these 433 clients have been reported in earlier studies $[10,11]$.

The clients were asked to rate their own perceived HIV risk in the questionnaire. Risk perception was stratified into four groups: "low risk" (<25\% chance), "medium risk" (25$75 \%$ chance), "high risk" (>75\% chance), and "unsure risk." Information regarding the clients' sexual behaviors, preferred role during anal sex, and other risk factors such as multiple sexual partners, types of sexual partners, alcohol use before sex, history of sexually transmitted infections (STIs), last unprotected sex, and condom use during anal sex, all in the preceding six months, was collated. Rapid test for HIV infection was performed using either the SD Bioline HIV test (Standard Diagnostics Inc.) or the ACON HIV test kits according to the manufacturer's specification.

Descriptive analysis was used for description of the demographic characteristics, sexual behaviors, and risk factors for acquisition of HIV infection. Chi-square analysis was used to determine the odds ratio of HIV acquisition in each of the risk groups. Regressions analysis was used to determine which of the risk factors were predictors of HIV acquisition in the low, medium, high, and unsure risk perceptions groups. All statistical analyses were performed using SPSS for Windows v21. A $P$ value of $<0.05$ with $95 \%$ confidence interval was deemed significant. This study received approval from the National Medical Research Register of Malaysia.

\section{Results}

3.1. Demographic Characteristics. Four hundred twentythree clients rated their own HIV risk perception in this study of which $350(82.7 \%)$ identified themselves as homosexuals while $73(17.3 \%)$ as bisexuals. The mean age was 29.2 years old. The youngest client was 18 years old and the oldest was 61 years old. Most of the clients were Chinese $(N=$ 250) followed by Malays $(N=115)$. Most had received tertiary education $(N=263)$ and earned a monthly income between MYR 2001 and $5000(N=152)$. The details of the demographic parameters are shown in Table 1.

Two hundred fifty-six clients (60.5\%) reported receiving VCT services at the same center previously prior to the survey. The breakdown according to risk categories was: low risk-64.4\%, medium risk $-67.8 \%$, high risk-77.8\%, and unsure risk-49.4\%, respectively.

3.2. HIV Risk Perception. From a total of 423 clients, 101 $(23.9 \%)$ clients perceived themselves to be at low risk $(<25 \%)$ of being infected with HIV at the time of survey, 118 (27.9\%) perceived themselves to be at medium risk (25-75\%), $36(8.5 \%)$ clients perceived themselves to be at high risk (>75\%), while the remaining 168 (39.7\%) clients were unsure regarding their risk.

3.3. Sexual Behavior and Preferred Role during Sex. Four hundred fifteen clients disclosed their sexual behaviors in the preceding 6 months. Most had engaged in anal and oral sex $(N=328,79.0 \%), 25(6.0 \%)$ had vaginal, anal, and oral sex, 23 (5.5\%) had anal sex only, 22 (5.3\%) had oral sex only, 9 (2.2\%) had vaginal and oral sex only, $6(1.4 \%)$ had vaginal sex only, and $2(0.5 \%)$ had vaginal and anal sex only (Table 1$)$. Out of 287 clients who disclosed their preferred sexual role during anal sex, 140 (48.8\%) were "versatile," 72 (25.1\%) were "top," and 75 (26.1\%) were "bottom" (Table 1).

3.4. Other Risk Factors for HIV Acquisition. Table 2 shows the number of clients in each risk perception group who had exposure to risk factors for HIV transmission in the preceding 6 months. The risk factors were multiple sexual partners (10 or less versus $>10$ ), promiscuity (regular versus regular and casual/transactional sex partners), alcohol use before sex, history of STIs, last unprotected sex, and condom use during anal sex.

An item in the questionnaire explored drug use before or during sex in the preceding 6 months. Out of 411 clients who responded to this question, only $42(10.2 \%)$ clients reported drug use before or during sex. As the number of respondents was small, meaningful conclusions using statistical methods could not be made.

3.5. Odds Ratio. Assigning clients who rated themselves to be at "low risk" to acquire HIV infection as the reference group, we determined the odds ratio for testing HIV positive in each of the other risk groups (i.e., medium risk, high risk, and unsure risk) for each of the risk factors for HIV transmission. The results are shown in Table 3.

The odds of testing positive for HIV infection in clients who rated themselves to be at "high risk" were $17 x$ higher compared to clients in the "low risk" group (OR 17.14, 95\% CI: $3.28-89.72, P<0.001)$ while clients who rated themselves to be at "medium risk" had 2.6x the odds of testing positive for HIV compared to "low risk" clients (OR 2.61, 95\% CI: 0.51$13.41, P=0.251)$. Clients who were unsure of their risk had $1.9 x$ the odds of testing HIV positive compared to "low risk" clients (OR 1.96, 95\% CI: $0.40-9.75, P=0.410$ ). The increased odds in the "medium risk" and "unsure risk" groups were not statistically significant compared to the "low risk" group.

Risk factors which were significant predictors for HIV acquisition in the "high risk" clients include having more than 10 sexual partners in the preceding 6 months, having casual and/or transactional sex partners in the preceding 6 months, 
TABLE 1: Demographic characteristics and sexual behavior of MSM clients.

\begin{tabular}{|c|c|c|c|c|}
\hline \multirow{2}{*}{ Demography and sexual behavior } & \multicolumn{4}{|c|}{ Perceived risk } \\
\hline & Low & Medium & High & Unsure \\
\hline \multicolumn{5}{|l|}{ (1) Age group in years $(N=421)^{*}$} \\
\hline$<20$ & $3(42.9 \%)$ & 0 & 0 & $4(57.1 \%)$ \\
\hline $20-29$ & $50(19.7 \%)$ & $78(30.7 \%)$ & $23(9.1 \%)$ & $103(40.6 \%)$ \\
\hline $30-39$ & $29(22.8 \%)$ & $33(26.0 \%)$ & $12(9.4 \%)$ & $53(41.7 \%)$ \\
\hline $40-49$ & $11(44.0 \%)$ & $6(24.0 \%)$ & $1(4.0 \%)$ & $7(28.0 \%)$ \\
\hline $50-59$ & $4(66.7 \%)$ & $1(16.7 \%)$ & 0 & $1(16.7 \%)$ \\
\hline$>60$ & $2(100 \%)$ & 0 & 0 & 0 \\
\hline \multicolumn{5}{|l|}{ (2) Ethnic group $(N=419)^{*}$} \\
\hline Malay & $15(13.0 \%)$ & $30(26.1 \%)$ & $11(9.6 \%)$ & $59(51.3 \%)$ \\
\hline Chinese & $64(25.6 \%)$ & $75(30.0 \%)$ & $20(8.0 \%)$ & $91(36.4 \%)$ \\
\hline Indian & $6(31.6 \%)$ & $3(15.8 \%)$ & 0 & $10(52.6 \%)$ \\
\hline Other & $15(42.9 \%)$ & $10(28.6 \%)$ & $3(8.6 \%)$ & $7(20.0 \%)$ \\
\hline \multicolumn{5}{|l|}{ (3) Sexuality $(N=423)^{*}$} \\
\hline Homosexual & $82(23.4 \%)$ & $101(28.9 \%)$ & $33(9.4 \%)$ & $134(38.3 \%)$ \\
\hline Bisexual & $19(26.0 \%)$ & $18(23.3 \%)$ & $3(4.1 \%)$ & $34(46.6 \%)$ \\
\hline \multicolumn{5}{|l|}{ (4) Education level $(N=353)^{*}$} \\
\hline Primary & $4(25.0 \%)$ & $2(12.5 \%)$ & 0 & $10(62.5 \%)$ \\
\hline Secondary & $19(25.7 \%)$ & $23(31.1 \%)$ & $5(6.8 \%)$ & $27(36.5 \%)$ \\
\hline Tertiary & $67(25.5 \%)$ & $78(28.7 \%)$ & $23(8.7 \%)$ & $95(36.1 \%)$ \\
\hline \multicolumn{5}{|c|}{ (5) Monthly income (MYR) $(N=394)^{*}$} \\
\hline$<1000$ & $6(20.0 \%)$ & $9(30.0 \%)$ & $4(13.3 \%)$ & $11(36.7 \%)$ \\
\hline $1000-2000$ & $15(16.9 \%)$ & $27(30.3 \%)$ & $10(11.2 \%)$ & $37(41.6 \%)$ \\
\hline $2001-5000$ & $37(24.3 \%)$ & $44(28.9 \%)$ & $16(10.5 \%)$ & $55(36.2 \%)$ \\
\hline$>5000$ & $16(39.0 \%)$ & $11(26.8 \%)$ & $3(7.3 \%)$ & $11(26.8 \%)$ \\
\hline \multicolumn{5}{|l|}{ (6) Sexual preference $(N=415)^{*}$} \\
\hline Anal and oral & $71(21.6 \%)$ & $98(28.8 \%)$ & $32(9.7 \%)$ & $128(38.9 \%)$ \\
\hline Oral only & $9(40.9 \%)$ & $5(22.7 \%)$ & 0 & $8(36.4 \%)$ \\
\hline Vaginal and anal and oral & $6(24.0 \%)$ & $6(24.0 \%)$ & $1(4.0 \%)$ & $12(48.0 \%)$ \\
\hline Anal only & $6(26.1 \%)$ & $4(17.4 \%)$ & $2(8.7 \%)$ & $11(47.8 \%)$ \\
\hline Vaginal only & $4(66.7 \%)$ & $1(16.7 \%)$ & 0 & $1(16.7 \%)$ \\
\hline Vaginal and oral & $3(33.3 \%)$ & $1(11.1 \%)$ & 0 & $5(55.5 \%)$ \\
\hline Vaginal and anal & 0 & $2(100 \%)$ & 0 & 0 \\
\hline \multicolumn{5}{|l|}{ (7) Role during anal sex $(N=287)^{*}$} \\
\hline Bottom & $11(14.7 \%)$ & $18(24.0 \%)$ & $11(14.7 \%)$ & $35(46.7 \%)$ \\
\hline Top & $21(29.2 \%)$ & $21(29.2 \%)$ & $3(4.2 \%)$ & $27(37.3 \%)$ \\
\hline Versatile & $32(22.9 \%)$ & $42(30.0 \%)$ & $11(7.9 \%)$ & $55(38.3 \%)$ \\
\hline
\end{tabular}

${ }^{*}$ Number of clients who responded to this part of the pre-HIV testing questionnaire.

MYR: Malaysian ringgit.

alcohol use before sex in the preceding 6 months, unprotected sex of more than 6 months ago, and inconsistent condom use during anal sex in the preceding 6 months (all $P<0.05$ ). The same factors were not statistically significant predictors in the "medium risk" and "unsure risk" groups $(P>0.05)$. History of sexually transmitted infections was not a predictor for HIV acquisition in any of the risk groups in this study (Table 3).

\section{Discussion}

MSM who rated themselves to be at high risk for being infected with HIV in this study were significantly associated with high risk factors and were $17 x$ more likely to be infected with HIV compared to those who rated themselves to be at low risk. The significant predictors of HIV transmission in the high HIV risk perception group were multiple sex partners, promiscuity, alcohol use before sex, unprotected sexual intercourse, and inconsistent condom use during anal sex (Tables 2 and 3). In contrast, clients who have rated themselves to be at medium risk or were unsure of their risk were not significantly associated with these risk factors and had lower odds of being infected with HIV compared to clients who rated themselves as "low risk." These risk factors have been well documented to be independent risk factors for HIV transmission among MSM [12-14].

The findings suggest that the clients were aware of the degree of their own high risk behaviors which enabled them to 
TABLE 2: Risk factors and prevalence of HIV in each perceived risk group.

\begin{tabular}{|c|c|c|c|c|}
\hline \multirow{2}{*}{ Risk factors (preceding 6 months) } & \multicolumn{4}{|c|}{ Perceived risk } \\
\hline & Low & Medium & High & Unsure \\
\hline \multicolumn{5}{|l|}{ (1) Number of sex partners $(N=273)^{*}$} \\
\hline$\leq 10$ & $34(54.8 \%)$ & $46(61.3 \%)$ & $12(54.5 \%)$ & $64(56.1 \%)$ \\
\hline$>10$ & $28(45.2 \%)$ & $29(39.7 \%)$ & $10(45.5 \%)$ & $50(43.9 \%)$ \\
\hline \multicolumn{5}{|l|}{ (2) Types of sex partners $(N=398)^{*}$} \\
\hline Regular & $44(46.3 \%)$ & $33(28.9 \%)$ & $13(40.6 \%)$ & $66(42.0 \%)$ \\
\hline Regular and casual/transactional sex & $51(53.6 \%)$ & $81(71.1 \%)$ & $19(59.4 \%)$ & $91(58.0 \%)$ \\
\hline \multicolumn{5}{|l|}{ (3) Alcohol use before sex $(N=313)^{*}$} \\
\hline No & $31(40.8 \%)$ & $37(44.6 \%)$ & $14(53.8 \%)$ & $72(56.3 \%)$ \\
\hline Yes & $45(59.2 \%)$ & $46(55.4 \%)$ & $12(46.2 \%)$ & $56(43.4 \%)$ \\
\hline \multicolumn{5}{|c|}{ (4) History of sexually transmitted infections $(N=412)^{*}$} \\
\hline No & $74(75.5 \%)$ & $91(79.1 \%)$ & $24(68.6 \%)$ & $137(83.5 \%)$ \\
\hline Yes & $24(24.5 \%)$ & $24(20.9 \%)$ & $11(31.4 \%)$ & $27(16.5 \%)$ \\
\hline \multicolumn{5}{|l|}{ (5) Last unprotected sex $(N=423)^{*}$} \\
\hline$<6$ months ago & $49(48.5 \%)$ & $82(69.5 \%)$ & $27(75.0 \%)$ & $94(56.0 \%)$ \\
\hline$>6$ months ago & $52(51.5 \%)$ & $36(30.5 \%)$ & $9(25.0 \%)$ & $74(44.0 \%)$ \\
\hline \multicolumn{5}{|l|}{ (6) Condom use during anal sex $(N=363)^{*}$} \\
\hline Consistent & $25(32.1 \%)$ & $22(21.0 \%)$ & $3(8.6 \%)$ & $36(24.8 \%)$ \\
\hline Inconsistent & $53(67.9 \%)$ & $83(79.0 \%)$ & $32(91.4 \%)$ & $109(75.2 \%)$ \\
\hline \multicolumn{5}{|l|}{ (7) HIV result $(N=423)^{*}$} \\
\hline Negative & $97(96.0 \%)$ & $109(92.4 \%)$ & $25(69.4 \%)$ & 155 (92.3\%) \\
\hline Positive & $4(4.0 \%)$ & $9(7.6 \%)$ & $11(30.6 \%)$ & $13(7.7 \%)$ \\
\hline
\end{tabular}

${ }^{*}$ Number of clients who responded to this part of the pre-HIV testing questionnaire.

TABLE 3: Odds of testing HIV positive in each perceived risk group for each of the risk factors using "low risk" as reference.

\begin{tabular}{|c|c|c|c|}
\hline Risk factor & Odds ratio & $P$ value & $95 \% \mathrm{CI}^{*}$ \\
\hline \multicolumn{4}{|c|}{ (1) $>10$ Sex partners in preceding 6 months } \\
\hline Medium risk & 1.364 & 0.761 & $0.18-10.06$ \\
\hline High Risk & 42.00 & $<0.001$ & $8.83-258.47$ \\
\hline Unsure risk & 1.475 & 0.676 & $0.24-9.15$ \\
\hline \multicolumn{4}{|c|}{ (2) Promiscuity (had > than regular sex partner) in preceding 6 months } \\
\hline Medium risk & 2.002 & 0.142 & $0.60-6.71$ \\
\hline High Risk & 10.670 & 0.019 & $3.13-36.36$ \\
\hline Unsure risk & 2.034 & 0.465 & $0.65-6.42$ \\
\hline \multicolumn{4}{|c|}{ (3) Alcohol use before sex in preceding 6 months } \\
\hline Medium risk & 2.002 & 0.613 & $0.60-6.71$ \\
\hline High Risk & 10.670 & $<0.001$ & $3.13-36.36$ \\
\hline Unsure risk & 0.041 & 0.928 & $0.65-6.42$ \\
\hline \multicolumn{4}{|c|}{ (4) History of sexually transmitted infections } \\
\hline Medium risk & 1.995 & 0.156 & $0.60-6.69$ \\
\hline High Risk & 10.771 & 0.261 & $3.15-36.81$ \\
\hline Unsure risk & 1.855 & 0.800 & $0.60-5.92$ \\
\hline \multicolumn{4}{|c|}{ (5) Last unprotected sex $>6$ months ago } \\
\hline Medium risk & 2.002 & 0.185 & $0.60-6.71$ \\
\hline High Risk & 10.670 & $<0.001$ & $3.12-36.36$ \\
\hline Unsure risk & 2.034 & 0.505 & $0.65-6.42$ \\
\hline \multicolumn{4}{|c|}{ (6) Inconsistent condom use during anal sex in preceding 6 months } \\
\hline Medium risk & 3.259 & 0.527 & $1.05-10.09$ \\
\hline High Risk & 12.182 & $<0.001$ & $3.80-39.03$ \\
\hline Unsure risk & 2.412 & 0.712 & $0.78-7.42$ \\
\hline
\end{tabular}

${ }^{*}$ CI: confidence interval up to 2 -decimal point. 
properly rate themselves into each of the HIV risk perception categories. This is in contrast to other studies among MSM that have shown that misperception of risk is the usual finding $[1,3-5]$. About $60 \%$ of the clients in our study had previously used the VCT services at the same center prior to the survey. These clients would most likely have received the prerequisite pre- and posttest counseling previously which may have contributed to greater awareness of their own risk factors. This may account for the clients' ability to rate their perceived risk fairly accurately.

Nevertheless, clients who did not rate themselves to be at "high risk" of HIV infection also reported considerable risk behaviors with similar proportions. The only exception was that of inconsistent condom use during anal sex with $91.4 \%$ of clients in the high HIV risk group compared to $67.9 \%$, $75.2 \%$, and $79 \%$ in the low, medium, and unsure risk groups, respectively (Table 2 ).

Surprisingly, history of STI was not a significant predictor for HIV transmission in any of the risk perception groups in our study. We believe this may be due to the structure and the anonymous nature of the questionnaire used in our study where detailed information regarding the types of STI and therapy received could not be readily obtained nor verified. Most clients were disinclined to reveal more information regarding the STI which they have had. Similarly, drug use is a legal offense in Malaysia and is punishable by law with either prolonged incarceration or capital punishment. This may have led to the reluctance of the clients to honestly disclose history of drug use before or during sexual intercourse making it difficult to interpret the available data meaningfully.

We recognized several major themes that have emerged from the findings in our study, namely, (1) MSM in this study were able to accurately rate their perceived risk of HIV infection which suggests they were aware of their individual risk factors; (2) the heightened awareness of the risk factors may have been contributed in part by previous counseling sessions these clients have received; (3) MSM in the high HIV risk perception group were associated with significant risk predictors of HIV transmission and were at much higher odds testing HIV positive compared to clients in the "low risk" category; and (4) despite rating themselves into lower risk groups, the MSM in these groups reported considerable risk behaviors suggesting the problem of misperceptions does exist to a certain degree in this study population.

This study has limited generalizability because it was confined to MSM who utilized the VCT services in one center and, therefore, may not be applicable to MSM who do not use VCT services. Nevertheless, in the setting of limited funding and resources, the findings in this study suggest that focused strategies to educate MSM regarding HIV and STI risks, especially in the high HIV risk perception group and efforts to raise personal risk perceptions, are critical because self-perception often guides the individual's testing and preventive behaviors [2]. Because some of the clients who reported lower risk perceptions in this study were found to be HIV-infected; consistent condom use during sexual intercourse regardless of the self-perceived risk with or without other risk factors should be the primary message to MSM during every VCT encounter.
In conclusion, MSM in this study could rate their HIV risk with reasonable accuracy. Perceived high HIV risk was associated with many of the established risk factors for transmission of HIV and STI. High risk perception was positively correlated with HIV infection. Strategies designed to change the sexual practices among those who perceived themselves to be at high risk of being infected with HIV should be put in place in a limited-resource setting.

\section{Conflict of Interests}

The authors declare that there is no conflict of interests regarding the publication of this paper.

\section{Acknowledgment}

The authors thank the PT Foundation, Malaysia, for their support and permission to use data collected from their voluntary counseling and testing program.

\section{References}

[1] M. J. Mimiaga, S. L. Reisner, E. F. Closson et al., "Self-perceived HIV risk and the use of risk reduction strategies among men who engage in transactional sex with other men in Ho Chi Minh City, Vietnam," AIDS Care, vol. 25, no. 8, pp. 1039-1044, 2013.

[2] K. H. Mayer, R. Ducharme, N. D. Zaller et al., "Unprotected sex, underestimated risk, undiagnosed HIV and sexually transmitted diseases among men who have sex with men accessing testing services in a New England bathhouse," Journal of Acquired Immune Deficiency Syndromes, vol. 59, no. 2, pp. 194198, 2012.

[3] CDC, "Unrecognized HIV infection, risk behaviors, and perceptions of risk among young black men who have sex with men-six U.S. Cities, 1994-1998," Morbidity and Mortality Weekly Report, vol. 51, no. 33, pp. 733-736, 1994.

[4] D. A. MacKellar, L. A. Valleroy, G. M. Secura et al., "Perceptions of lifetime risk and actual risk for acquiring HIV among young men who have sex with men," AIDS and Behavior, vol. 11, no. 2, pp. 263-270, 2007.

[5] W. Ma, X. Ding, H. Lu et al., "HIV risk perception among men who have sex with men in two municipalities of Chinaimplications for education and intervention," AIDS Care, vol. 25, no. 3, pp. 385-389, 2013.

[6] S. Baral, F. Sifakis, F. Cleghorn, and C. Beyrer, "Elevated risk for HIV infection among men who have sex with men in lowand middle-income countries 2000-2006: a systematic review," PLoS Medicine, vol. 4, no. 12, pp. 1901-1911, 2007.

[7] USAID Health Policy Initiative, HIV Expenditure on MSM Programming in the Asia-Pacific Region, Washington, DC, USA, 2006.

[8] W. D. Johnson, D. R. Holtgrave, W. M. McClellan, W. D. Flanders, A. N. Hill, and M. Goodman, "HIV intervention research for men who have sex with men: a 7-year update," AIDS Education and Prevention, vol. 17, no. 6, pp. 568-589, 2005.

[9] J. H. Herbst, R. T. Sherba, N. Crepaz et al., "A meta-analytic review of HIV behavioral interventions for reducing sexual risk behavior of men who have sex with men," Journal of Acquired Immune Deficiency Syndromes, vol. 39, no. 2, pp. 228-241, 2005. 
[10] K. C. Koh and A. Kamarulzaman, "Profiles of men-who-havesex-with-men seeking anonymous voluntary HIV counseling and testing at a community-based centre in Malaysia," Medical Journal of Malaysia, vol. 66, no. 5, pp. 491-494, 2011.

[11] K. C. Koh, K. Kanagalingam, F. T. Tai, and A. Kamarulzaman, "Sexual practices and HIV prevalence amongst men who have sex with men at a community-based voluntary counseling and testing centre in Malaysia," ISRN Infectious Diseases, vol. 2013, Article ID 247545, 6 pages, 2013.

[12] M. M. Silvia, L. S. Jose, L. Alberto et al., "Prevalences, genotypes, and risk factors for HIV transmission in South America," Journal of Acquired Immune Deficiency Syndromes, vol. 40, no. 1, Article ID 57064, pp. 57-64, 2005.

[13] M. T. Schechter, W. J. Boyko, B. Douglas et al., “The Vancouver lymphadenopathy-AIDS study: 6 HIV seroconversion in a cohort of homosexual men," Canadian Medical Association Journal, vol. 135, no. 12, pp. 1355-1360, 1986.

[14] J. L. Burcham, B. Tindall, M. Marmor, D. A. Cooper, G. Berry, and R. Penny, "Incidence and risk factors for human immunodeficiency virus seroconversion in a cohort of Sydney homosexual men," Medical Journal of Australia, vol. 150, no. 11, pp. 634-639, 1989. 


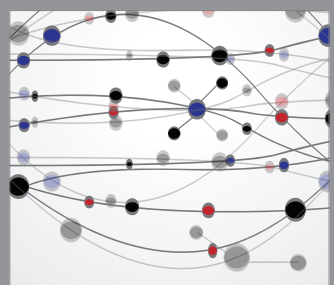

The Scientific World Journal
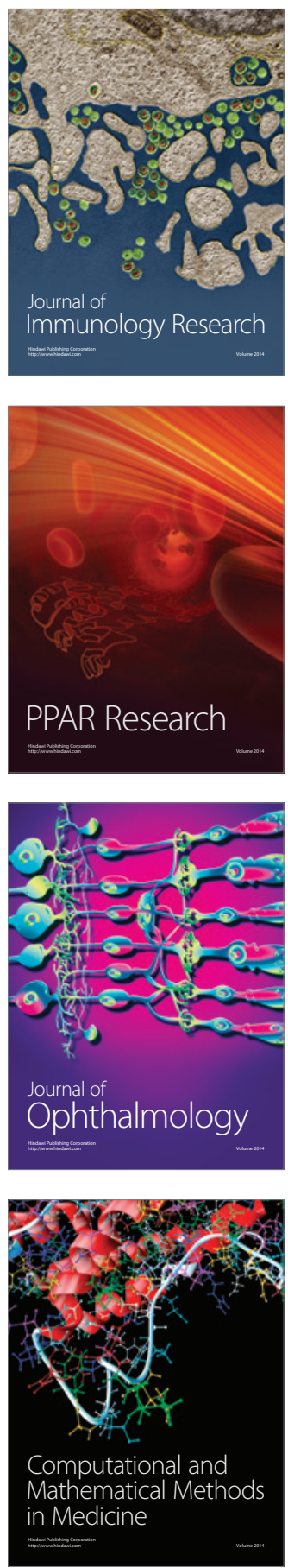

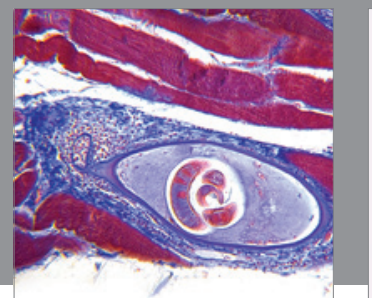

Gastroenterology

Research and Practice
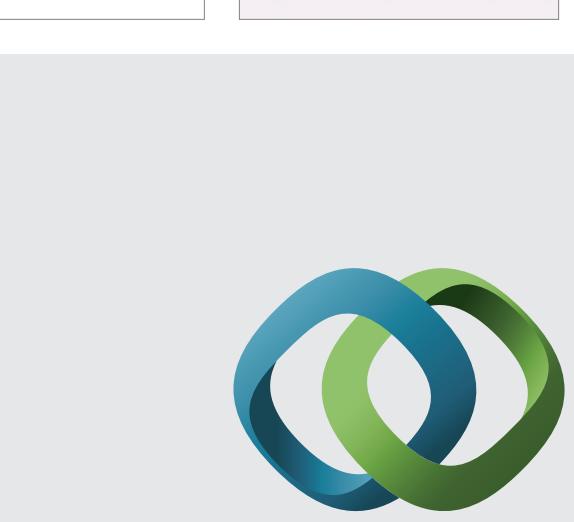

\section{Hindawi}

Submit your manuscripts at

http://www.hindawi.com
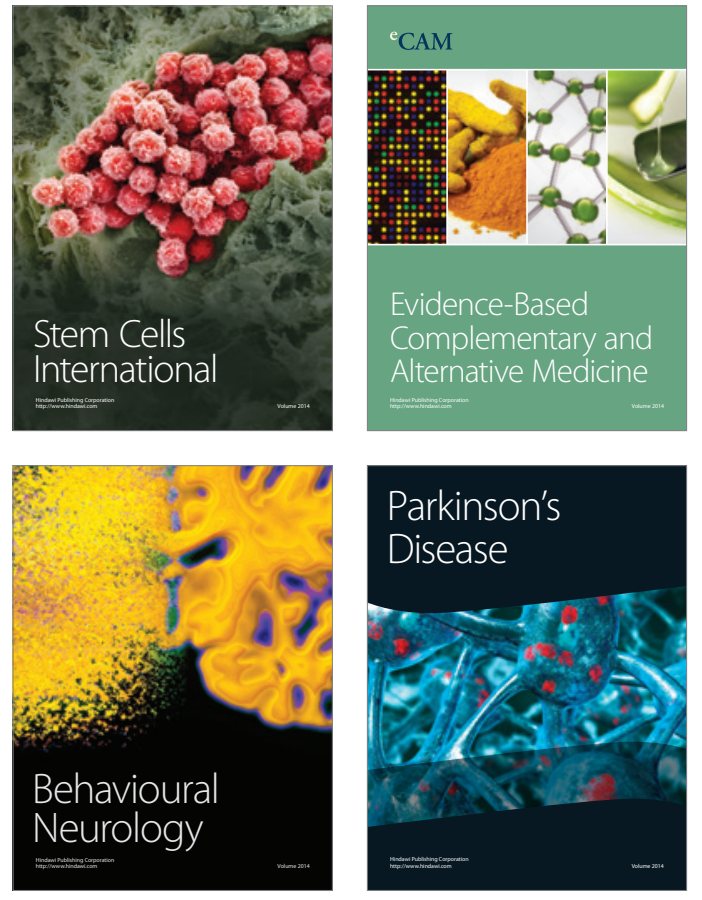
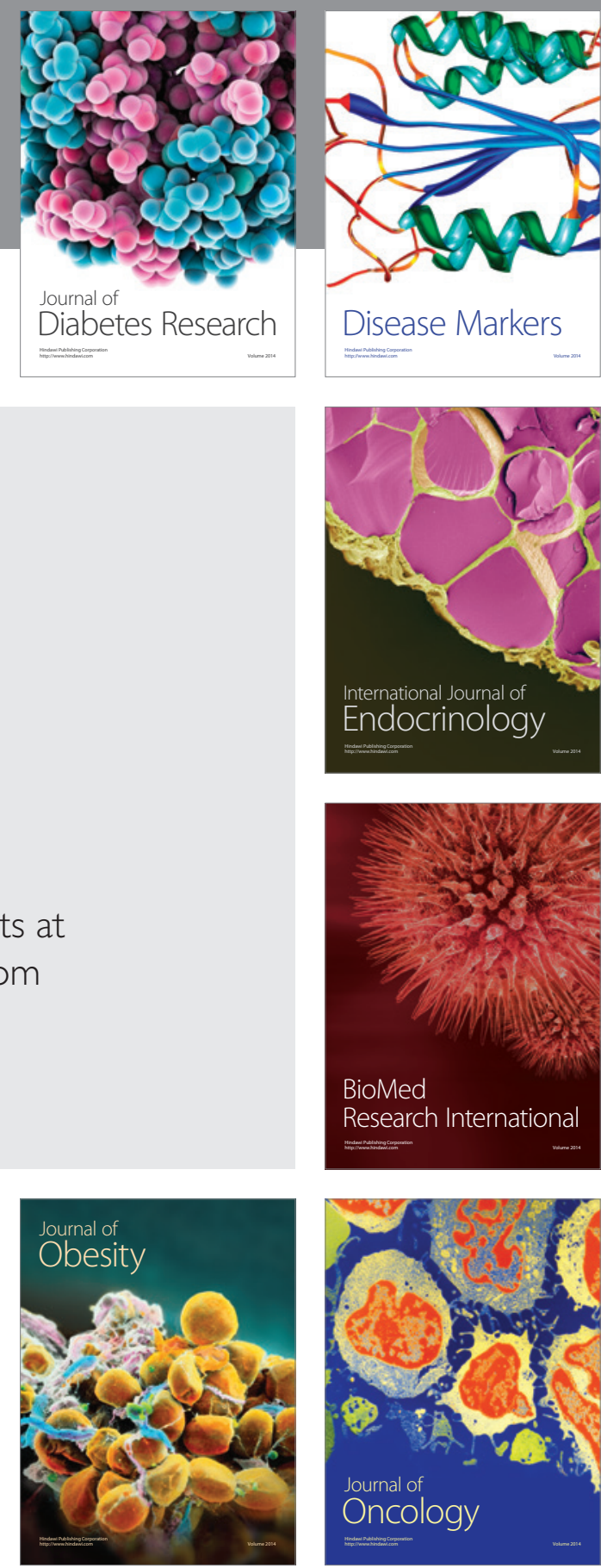

Disease Markers
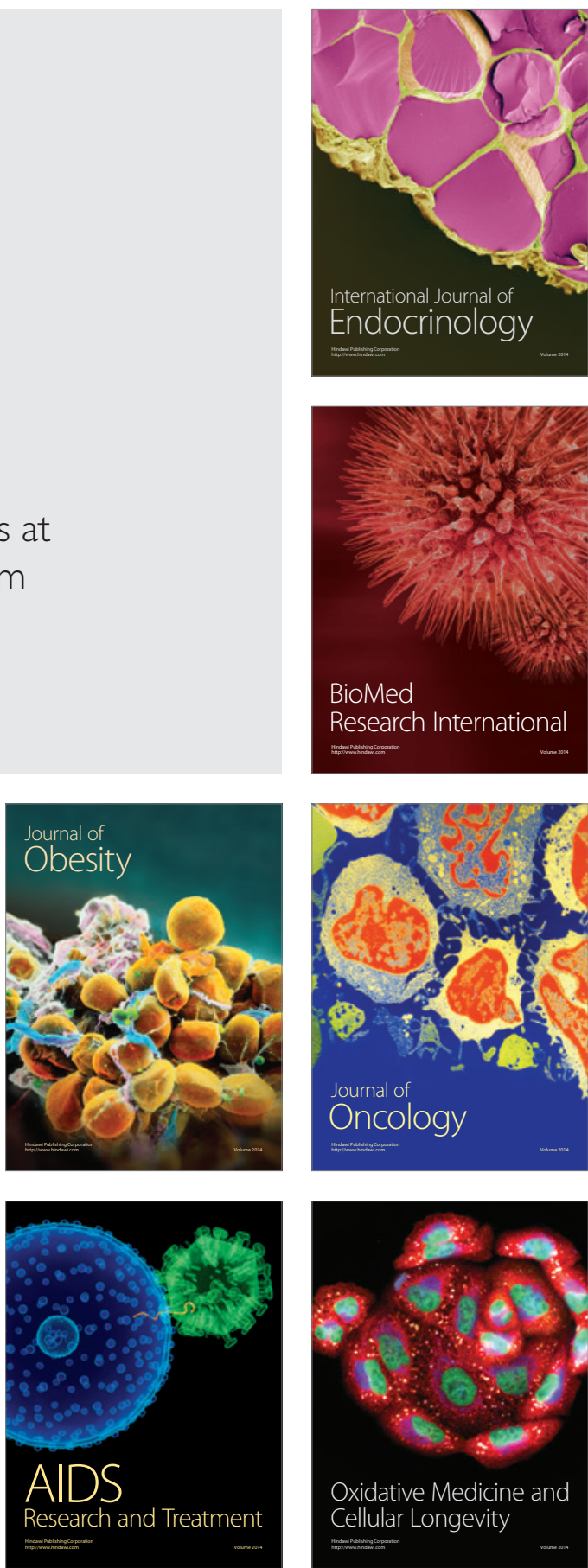